\title{
KINETIC ANALYSIS OF SEVERAL VARIATIONS OF PUSH-UPS
}

\author{
Bradley Wurm ${ }^{1}$, Tyler L. VanderZanden ${ }^{1}$, Mark Spadavecchia ${ }^{1}$, John Durocher ${ }^{2}$, Curtis \\ Bickham ${ }^{3}$, Erich J. Petushek ${ }^{4}$, and William P. Ebben ${ }^{1,3}$
}

Department of Physical Therapy, Program in Exercise Science, Strength and Conditioning Research Laboratory, Marquette University, Milwaukee, WI, USA ${ }^{1}$ Department of Physical Therapy, St. Francis University, Loretto, PA, USA ${ }^{2}$ Department of Health, Exercise Science \& Sport Management, University of Wisconsin-Parkside, Kenosha, WI, USA ${ }^{3}$

Department of Health Physical Education and Recreation, Northern Michigan University, Marquette, MI, USA ${ }^{4}$

\begin{abstract}
Push-ups are a common and practical exercise though the kinetic characteristics of this exercise and its variations have yet to be quantified. This study assessed the peak ground reaction forces (GRF) of push-up variations including the regular push-up and those performed with bent knee, feet elevated on a $30.48 \mathrm{~cm}$ box and a $60.96 \mathrm{~cm}$ box, hands elevated on a $30.48 \mathrm{~cm}$ box and a $60.96 \mathrm{~cm}$ box. Peak GRF and peak GRF expressed as a coefficient of subject body mass were obtained with a force platform. Push-ups with the feet elevated produced higher GRF than all other push-up variations $(p \leq 0.05)$. Push-ups with hands elevated and from the bent knee position produced lower GRF than all other push-up variations $(p \leq 0.05)$. These data can be used to progress the intensity of pushups in a program with loads that are quantified as a percentage of body mass.
\end{abstract}

KEYWORDS: strength, body weight, upper body, closed kinetic chain

INTRODUCTION: Push-ups are a commonly performed, practical, easy to execute, multijoint upper body exercise that do not require expensive equipment. Push-ups have been recommended as one of the best practical upper body exercises in a popular consumer publication (Lee, 2008). Push-ups are one of a limited number of closed kinetic upper body exercises (Blackard et al., 1999) and there are many potential variations (Osbourne, 1989). Unfortunately, the quantification of this exercise stimulus in a training program is difficult compared to more traditional resistance training exercises. Resistance training exercises are often performed with equipment such as a barbell and weight plates with clearly labeled masses. These loads are often calculated and exercises are performed with a percentage of the exercisers maximum ability. Determination of the intensity of a resistance training stimulus allows for the progression of exercise intensity and the calculation of training volume.

Push-ups have also been evaluated as an upper body strength test (Mayhew et al., 1991) and have been demonstrated to increase upper body strength and power (Vossen et al., 2000). Research quantifying push-ups and the variations of this exercise is limited. The traditional push-up has been compared to a manufactured product purported to increase the quality of the push-up training stimulus using electromyography and video analysis to assess the differences (Bohne, et al., 2009). Research using electromyography to assess muscle activation demonstrated that variations in hand stance width resulted in different levels of muscle activation of the pectoralis major and triceps brachii (Cogley et al., 2005). Only one study used ground reaction forces (GRF), as well as electromyography, to assess variations in push-ups characterized by differences in hand position as well as a bent knee condition. This study described the push-up as a percentage of body weight and demonstrated differences in GRF between variations of hand placement and between bent knee and normal push-up conditions (Gouvali \& Boudolos, 2005). Many variations of pushups have yet to be studied including the conditions where feet are elevated or where hands are elevated. The purpose of this study was to assess the GRF associated with regular push-ups, and those performed with bent knee, feet elevated on a 12" box, feet elevated on 24" box, hands elevated on a 12" box, and hands elevated on a 24" box for the purpose of quantifying the intensity of these exercises for exercise progression and to allow for the 
calculation of exercise load and volume in a program. This study also sought to assess if there were gender based differences in response to these push-up variations.

METHODS: Twenty-three recreationally fit young adults (mean \pm SD; age $=22.5 \pm 4.6$ years, height $=178.10 \pm 10.89 \mathrm{~cm}$; body mass $=80.59 \pm 9.28 \mathrm{~kg}$ ) volunteered to serve as subjects for the study. Subjects signed an informed consent form and Institutional Review Board approval was obtained prior to the study.

Subjects were instructed in and received demonstration of each push-up variation including a regular push-up, and those performed with bent knee, feet elevated on a $30.48 \mathrm{~cm}$ box, feet elevated on $60.96 \mathrm{~cm}$ box, hands elevated on a $30.48 \mathrm{~cm}$ box, and hands elevated on a $60.96 \mathrm{~cm}$ box. For all warm up, practice and test push-ups, subjects hand placement was defined as the width equal to the distance of contralateral acromion processes measured from the inside border of each hand with hands placed under the shoulders in the beginning position which was characterized by full elbow extension.

Subjects warmed-up by performing 3 practice repetitions of each push-up variation in a randomized order. Subjects then rested for 2 minutes. Subjects then performed 2 repetitions of each push-up variation in randomized order. Subjects rested for 1 minute in between each push-up variation. A metronome was used to control the cadence of the push-up repetitions with each repetition performed for a count of two seconds. A fast pace was desired since previous research demonstrated higher levels of power and work for fast compared to slower cadences (LaChance and Hortobgyi,1994) and the 2 second cadence has been demonstrated to be effective at increasing upper body strength (Vossen, et al., 2000). The push-up variations were assessed with a $60 \times 120 \mathrm{~cm}$ force platform (BP6001200, Advanced Mechanical Technologies, Inc., Watertown, MA, USA), which was calibrated with known loads to the voltage recorded prior to the testing session. Data were collected at 1000 $\mathrm{Hz}$, real time displayed and saved with the use of computer software (BioAnalysis 3.1, Advanced Mechanical Technologies, Inc., Watertown, MA, USA) for later analysis. All values were determined as the average of two trials for each push-up variation. Peak GRF and peak GRF expressed as percentage of static body mass GRF were evaluated from the vertical force-time records using custom designed software. Data were analyzed using SPSS 16.0 with a repeated measures ANOVA to determine possible differences between push-up variations. Bonferroni adjusted pairwise comparisons identified the specific differences between these variations. The a priori alpha level was set at $p \leq 0.05$. Effect sizes and power are reported as $\eta_{\mathrm{p}}{ }^{2}$ and $d$, respectively.

RESULTS: There was a significant main effects for push-up condition for body weight coefficient $\left(p \leq 0.001, \eta_{\mathrm{p}}{ }^{2}=0.94, d=1.00\right)$, with no interaction between push-up condition and gender for this variable $(p=0.25)$. There was a significant main effect for peak GRF ( $p$ $\left.\leq 0.001, \eta_{\mathrm{p}}{ }^{2}=0.76, d=1.00\right)$ with no interaction between condition and gender for this variable $(p \leq 0.05)$. Trial to trial reliability of the body weight coefficient were moderately to highly reliable as demonstrated by Intraclass Correlation Coefficient values in a range from 0.64 to 0.84 with no significant differences between trials $(p>0.05)$. Trial to trial reliability of the mean peak GRF were highly reliable as demonstrated by Intraclass Correlation Coefficient values in range from 0.97 to 0.99 with no significant differences between trials ( $p$ $>0.05$ ). 
Table 1. Push up peak ground reaction force expressed as a percentage of subject static body mass peak ground reaction force.

\begin{tabular}{lc}
\hline Push-Up Variation & Body Weight Coefficient \\
\hline Feet Elevated $60.96 \mathrm{~cm}$ & $0.74 \pm 0.02^{\star}$ \\
Feet Elevated $30.48 \mathrm{~cm}$ & $0.70 \pm 0.02^{\star}$ \\
Regular & $0.64 \pm 0.04^{\star}$ \\
Hands Elevated $30.48 \mathrm{~cm}$ & $0.55 \pm 0.05^{\star}$ \\
Bent Knee & $0.49 \pm 0.05^{\star}$ \\
Hands Elevated $60.96 \mathrm{~cm}$ & $0.41 \pm 0.06^{\star}$ \\
\hline
\end{tabular}

*Significantly different than all other push up conditions $(p \leq 0.01)$

Table 2. Push up peak ground reaction expressed in Newtons (N) for each push up variation.

\begin{tabular}{lc}
\hline Push-Up Variation & Peak GRF \\
\hline Feet Elevated $60.96 \mathrm{~cm}$ & $723.89 \pm 138.24^{*}$ \\
Feet Elevated $30.48 \mathrm{~cm}$ & $714.05 \pm 142.61^{\pi}$ \\
Regular & $700.13 \pm 138.25^{\star}$ \\
Hands Elevated $30.48 \mathrm{~cm}$ & $681.46 \pm 125.31^{\star}$ \\
Hands Elevated $60.96 \mathrm{~cm}$ & $596.75 \pm 113.43^{\star}$ \\
Bent Knee & $569.92 \pm 138.24^{\star}$
\end{tabular}

*Significantly different $(p \leq 0.05)$ than all other push up conditions.

"Significantly different $(p \leq 0.05)$ than all other push up conditions except Feet Elevated $60.96 \mathrm{~cm}$

* Significantly different $(p \leq 0.05)$ than all other push up conditions except Feet Elevated $30.48 \mathrm{~cm}$

DISCUSSION: This is the first study to assess push-up variations that include varying levels of feet and hand elevation, demonstrating differences between these conditions. Results of this study can be used to guide the progression of overload, by incorporating the push-up variations with higher GRF's over time. Progression of overload is believed to be important for exercise program design (Fleck \& Kraemer, 1997).

Results of this study demonstrated GRFs of approximately $64 \%$ and $49 \%$ of body weight in the regular and bent knee push-up conditions, respectively. These findings were slightly different than the values of $66 \%$ and $53 \%$, for the regular and bent knee push up variations, respectively, previously found (Gouvali \& Boudolos, 2005). Changes in hand placement produce GRFs in a range from $52.9 \%$ to $72.9 \%$ of body mass (Gouvali \& Boudolos, 2005), whereas the present study demonstrates that push-up conditions that included hand and feet elevation produced values that ranged from approximately $41 \%$ to $74 \%$ of body mass.

CONCLUSION: Practitioners should progress push-up intensity from lower intensity push-up variations such as the elevated hand and bent knee conditions to normal push-ups to the feet elevated conditions. These data can also be used to quantify the approximate load, as a percentage of body mass for the purpose of quantifying exerciser load and volume in a resistance training program.

\section{REFERENCES:}

Blackard, D.O., Jensen R.L. \& Ebben W.P. (1999). Use of EMG analysis in challenging kinetic chain terminology. Medicine and Science in Sport and Exercise. 31(3), 443-448.

Cogley, R.M., Archambault, T.E., Fiberger, J.F., Koverman, M.M., Youdas, J.M., \& Hollman, J.H. (2005). Comparison of muscle activation using various hand positions during the push up exercise. Journal of Strength and Conditioning Research. 19, 628-633.

Bohne, M., Slack, J., Claybaugh, T., \& Cowley, J. (2009) A comparison of the Perfect Push-Up ${ }^{\mathrm{TM}}$ to traditional pushup. In Proceedings of the XXVII Congress of the International Society of Biomechanics in Sports.

Fleck, S. J., \& Kraemer, W.J. (1997). Designing resistance training programs. Champaign, IL. Human Kinetics. pp. 7.

Gouvali, M.K., \& Boudolos, K. (2005) Dynamic and electromyographical analysis in variants of pushup exercise. Journal of Strength and Conditioning Research. 19(1), 146-151. 
LaChance, P.F., \& Hortobagyi, T. (1994) Influence of cadence on muscle performance during pushup and pull-up exercise. Journal of Strength and Conditioning Research. 8(2), 76-79.

Lee, J. (2008) A new way to do push-ups. Shape. 27(6), 132.

Mayhew, J.L., Ball, T.E., Arnold, M.D., \& Bowen, J.C. (1991) Push-ups as a measure of upper body strength. Journal Applied Sport Science Research, 5(1), 16-21.

Osbourne, R. (1989) Variations on the push-up. NSCA Journal. 11(1), 28-29.

Vossen, J.F., Kramer, J.F., Burke, D.G., \& Vossen, D.P. (2000) Comparison of dynamic push-up training and plyometric push-up training on upper-body power and strength. Journal of Strength Conditoning Research. 14(3), 248-253.

Acknowledgement

Travel to present this study was funded by a Green Bay Packers Foundation Grant. 